\title{
Salvia suspension cultures as production systems for oleanolic
}

\section{and ursolic acid}

This is the peer-reviewed version from research article Acta Physiologiae Plantarum, 36 (8), Christiane Haas, Karl-Christoph Hengelhaupt, Sibylle Kümmritz, Thomas Bley, Atanas Pavlov, Juliane Steingroewer, 2014, Salvia suspension cultures as production systems for oleanolic and ursolic acid, 2137-2147, which has been published in final form at: http://dx.doi.org/ $10.1007 / \mathrm{S} 11738-014-1590-0$ (c) Springer. Personal use of this material is permitted. Permission from publisher must be obtained for all other users. The final publication is available at Springer via the above mentioned link.

Christiane Haas $^{\mathrm{a}^{*}}$, Karl-Christoph Hengelhaupt ${ }^{\mathrm{a}}$, Sibylle Kümmritz ${ }^{\mathrm{a}}$, Thomas Bley ${ }^{\mathrm{a}}$, Atanas Pavlov $^{\mathrm{b}, \mathrm{c}}$, Juliane Steingroewer ${ }^{\mathrm{a}}$

${ }^{a}$ Institute of Food Technology and Bioprocess Engineering, Technische Universität Dresden, Bergstraße 120, 01069 Dresden, Germany

${ }^{\mathrm{b}}$ The Stephan Angeloff Institute of Microbiology, Bulgarian Academy of Sciences, 139

Ruski Blvd., 4000 Plovdiv, Bulgaria

${ }^{\mathrm{c}}$ Department of Organic Chemistry, University of Food Technologies, 26 Maritza Blvd, 4000 Plovdiv, Bulgaria

*corresponding author

Phone: 0049-351-463-32420

Fax: 0049-351-463-37761

Email: Christiane.Haas@mailbox.tu-dresden.de 


\begin{abstract}
Oleanolic and ursolic acid (OA and UA) are triterpenic acids with diverse biological activities that are of interest to the pharmaceutical industry. To investigate the scope for producing these compound using cell suspension cultures of Salvia species, calli from S. officinalis, S. virgata and S. fruticosa were induced using several plant growth regulator (PGR) combinations. Eleven lines were selected for suspension induction from a pool of calli. Six suspension cultures were established successfully and cultivated in the Respiration Activity MOnitoring System ${ }^{\circledR}\left(\right.$ RAMOS $\left.^{\circledR}\right)$ to obtain online data on their growth kinetics and to establish appropriate sampling schedules for the determination of their OA and UA production. Based on their observed growth behaviour, OA and UA contents, and aggregation properties, one suspension culture from each studied Salvia species was selected for further optimisation. The $\mu_{\max }$ values for these suspension cultures ranged from 0.20 to $0.37^{\circ} \mathrm{d}^{-1}$, their OA and UA contents were greater than 1.3 and $1.2 \mathrm{mg} \mathrm{g}^{-1}$, respectively, and they afforded maximum volumetric yields of $21.0 \mathrm{mg} \mathrm{l}^{-1}$ for $\mathrm{OA}$ and $32.8 \mathrm{mg}^{-1}$ for UA. These results will be useful in the development of a refined Salvia suspension-based process for OA and UA production.
\end{abstract}

Keywords: Salvia officinalis, Salvia fruticosa, Salvia virgata, callus, bioactive triterpene, RAMOS $^{\circledR}$ - respiration activity monitoring system

Abbreviations: AGG: area gained by growth; DW: dry weight; LS: Linsmaier and Skoog medium; MS: Murashige and Skoog medium; OA: oleanolic acid; OTR: oxygen transfer rate; PGR: plant growth regulator; RQ: respiration quotient; UA: ursolic acid; 


\section{Introduction}

Oleanolic acid (OA) and ursolic acid (UA) are triterpenic acids with diverse biological activities including hepato-protective and anticancer properties (Liu 2005). In China, they are used in the clinic to treat liver diseases such as hepatitis (Liu 1995). The development of a GMP-compliant process for producing these substances using plant cell suspension cultures could increase the scope for exploiting their application in the areas of medicine. Salvia species produce various monoterpenes or essential oils (Taarit et al. 2011) but some of them also produce OA and UA in amounts that can exceed $1 \%$ of their total dry weight (DW) (Martin et al. 2009; Janicsák et al. 2006), especially S. fruticosa, S. officinalis and S. virgata. The measured OA and UA contents of these species vary depending on their growth conditions, the variety used, and the analytical procedure used to perform the measurements. OA contents ranging from 0.001 to $2.0 \%(\mathrm{w} / \mathrm{w})$ have been measured while those for UA range from 0.4 to $3.8 \%(\mathrm{w} / \mathrm{w})$ (Table 1$)$.

At present, the literature contains relatively little data on callus or cell suspension cultures of Salvia species whose capacity for producing OA and/or UA has been tested (Georgiev et al. 2011; Bolta et al. 2000). S. officinalis cultures have been evaluated for the production of UA (Bolta et al. 2000). While a suspension culture of $S$. fruticosa has been reported, it was only evaluated for the production of rosmarinic acid (Karam et al. 2003; Kintzios et al. 1999). To the best of our knowledge, there have been no published studies on callus or suspension cultures of $S$. virgata.

Cultivation experiments using plant cell suspension cultures are often performed in standard shake flasks. This approach has a significant drawback in that the entire contents of one or more flasks must be harvested in order to characterise the culture's growth, which is very time consuming and laborious. The RAMOS ${ }^{\circledR}$ (Respiratory Activity MOnitoring System) eliminates this drawback by enabling on-line monitoring of the oxygen transfer rate (OTR) and carbon dioxide transfer rate (CTR) within individual shake 
flasks (Anderlei et al. 2004; Anderlei and Büchs 2001). The availability of this on-line information makes it possible to monitor the culture's growth on a regular basis without taking samples. Since its introduction the RAMOS has been widely used in the cultivation of microorganisms (Kottmeier et al. 2012; Kensy et al. 2009; Anderlei et al. 2004; Anderlei and Büchs 2001) and has been applied to plant in vitro cultures already (Geipel et al. 2013; Ullisch et al. 2012). Basic investigations into the adaption of the RAMOS for cultivation of plant cells were reported by Geipel et al. (2013). It can be challenging to screen various suspension cultures under different growth conditions or cultures that behaves very differently because the standard procedure is to sample all cultures on the same day of cultivation (Mathur and Shekhawat 2013), but the results obtained by doing this will not necessarily be comparable when dealing with cultures that have very different properties. For example, it is possible that a given time of sampling may correspond to the time at which the biomass concentration of one suspension is maximized whereas a different culture may have already passed its peak at the same point in time. One way of avoiding this problem is to sample narrowly (e.g. on a daily basis). However, this is very laborious. Alternatively, by using the RAMOS, one can monitor the growth kinetics of each culture continuously and decide when to collect punctual samples based on these data. The main aim of this study was to establish cell suspension cultures of Salvia species, study their growth and metabolic activity, and determine their potential for the production of OA and UA. A secondary objective was to evaluate RAMOS as a tool for screening plant cell suspension cultures.

\section{Material and methods}

\section{Callus cultures}

Seeds of the Salvia officinalis L. variety "Extrakta" purchased from N.L. Chrestensen Erfurter Samen- und Pflanzenzucht GmbH (Erfurt, Germany) and of Salvia virgata 
received from Hortus botanicus Hauniensis, University Copenhagen (Copenhagen, Denmark) were potted in common garden soil and grown in the lab. Callus formation was induced on leaves and leaf stems from young (3-9 weeks old) S. officinalis and S. virgata plants. The leaves used for this purpose were taken from the 2. - 4. position below the shoot tip. Callus formation from Salvia fruticosa syn. S. triloba was induced from leaves and leaf stems from a 2-year old plant that was ordered from Die Kräuterei (Oldenburg, Germany).

The surfaces of the plant explants were sterilised as described previously (Georgiev et al. 2011). The explants were cultivated in Murashige and Skoog (MS) medium including vitamins supplemented with $30 \mathrm{~g}^{-1}$ sucrose, $5.55 \mathrm{~g}^{-1}$ phyto-agar ( $\left.\mathrm{pH} 5.6-5.8\right)$ and various plant growth regulators (PGR's) in various combinations. The PGR's included the auxins 2,4-dichlorophenoxy acid (2,4-D), 1-naphthalene acetic acid (NAA), and picloram (Pic); and cytokinins represented by kinetin (Kin), 6-benzylaminopurine (BAP) and zeatin (Zea). Callus induction experiments were performed using each auxin in combination with each cytokinin and also by itself. The following auxin/cytokinin ratios at mass concentration ( $\mathrm{mg} \mathrm{l}^{-1}$ ) were used: $0.5 / 0.5,1 / 0.5,2 / 0.5,1 / 1,2 / 1$ and $2 / 2$. Experiments using only one auxin were performed with auxin concentrations of $0.2,0.5,1$ or $2 \mathrm{mg}^{-1}$. Calli from $S$. fruticosa were only induced on media containing PGR combinations with 2,4-D. All chemicals were purchased from Duchefa Biochemie BV (Haarlem, The Netherlands). Calli were sub-cultivated with three week intervals. Biomass samples were collected from the cultures, freeze dried and used for analysis of OA and UA 8 to 12 months after induction. The growth behaviour of the cultures was studied 14 months after induction (see the Analyses section for details).

\section{Suspension cultures}

Approximately 15 months after callus induction, eleven lines were selected for use in establishing suspension cultures (see Table 3, section Results and Discussion). The 
corresponding calli were transferred into liquid Linsmaier and Skoog (LS) media (including vitamins) that was supplemented with $30 \mathrm{~g} \mathrm{l}^{-1}$ sucrose and $0.2 \mathrm{mg} \mathrm{l}^{-1} 2,4-\mathrm{D}(\mathrm{pH}$ 5.6-5.8). Suspension cultures were maintained as described previously (Geipel et al. 2013). Sub-cultivation was performed between 8 and 11 days depending on the line being used and its rate of growth. Aggregates were shortly allowed to settle down before subcultivation to remove bigger aggregates $(>0.5 \mathrm{~cm})$ within the suspensions. After $6-8$ passages of sub-cultivation the suspension cultures were growing stably, which means stable growth behaviour and stable morphological characteristics. At least nine passages of sub-cultivation were performed before setting up RAMOS.

\section{RAMOS and shake flask cultivation}

The RAMOS (HiTec Zang, Herzogenrath, Germany) was used for the cultivation and online monitoring of the established suspension culture lines. The names of the individual lines and the growth conditions of their corresponding callus lines are shown with a grey background in Table 3. Cultivations were performed as described previously (Geipel et al. 2013). The inoculum volume was $20 \%(\mathrm{v} / \mathrm{v})$ of the working volume for all cultures than $\mathrm{S}_{\text {off_b }}$, for which it was $(40 \%, \mathrm{v} / \mathrm{v})$. Every suspension other than $\mathrm{S}_{\text {off_b }}$ and $\mathrm{S}_{\text {fru_a }}$ Was cultivated and measured in the RAMOS three times independently: twice at $110 \mathrm{rpm}$ and once at $150 \mathrm{rpm}$, with a shaking diameter $50 \mathrm{~mm}$ shaking for both shaking frequencies. The $S_{\text {off_b }}$ culture was measured only once at $110 \mathrm{rpm}$, while $\mathrm{S}_{\text {fru_a }}$ was measured twice at $110 \mathrm{rpm}$. Each cultivation was performed with two to four replicates.

The cultivation time varied depending on the culture's growth behaviour, and cultivation was halted when the OTR started to decline sharply. Sampling was conducted based on the on-line OTR data, at three separate time points: (1) at the start of the cultivation; (2) during the increasing OTR phase, which corresponds to the late growth phase; and (3) when the OTR stopped increasing. One entire shake flask was harvested per sample and processed as described in the Analyses section. 
To better understand the kinetics of product formation an additional shake flask experiment was performed using the $\mathrm{S}_{\text {off_a }}$ suspension culture, which was prepared in the same way as in the RAMOS experiments. Twenty shake flasks ( $250 \mathrm{ml}, 50 \mathrm{ml}$ working volume) were prepared, two of which were harvested on each day of sampling.

\section{Analyses}

\section{Callus growth}

The growth of callus was determined by measuring their area gained by growth (AGG) based on photos that were taken directly after sub-cultivation and three weeks later. The area occupied by each callus was determined by counting the number of pixels corresponding to the callus and the Petri dish using ImageJ (McMaster Biophotonics Facility, McMaster University, Hamilton, Canada). The number of pixels occupied by the callus was normalized against the number of pixels corresponding to the area of the Petri dish. The area of the Petri dish was defined using the oval selection tool. Areas that had been covered by the growth of the callus were identified by manually adjusting the image's hue and saturation and with the help of the wand tool. The 'area gained by growth' (AGG) was then calculated using the following equation:

$$
A G G=\left(\frac{\left(\frac{\text { area occupied by calli }[\text { pixel }]}{\text { area petri dish }[\text { pixel }]}\right)_{t=21[d]}}{\left(\frac{\text { area occupied by calli }[\text { pixel }]}{\text { area petri dish }[\text { pixel }]}\right)_{t=0[d]}}-1\right) \times 100 \%
$$

The relative standard deviation for AGG estimates performed with this method is $12.9 \%$ based on ten independent analyses of the same picture.

\section{Growth of suspensions}

The content of a whole shake flask was filtrated to isolate the biomass, which was transferred to a Petri dish. The wet weight (WW) and DW of the biomass were determined by measuring the material before and after freeze drying (Alpha 1-2 lyophilizer, Christ, 
Osterode am Harz, Germany). The WW and DW concentrations were calculated based on the initial used culture volume of $50 \mathrm{ml}$ in both cases in order to neglect the influence of $\mathrm{H}_{2} \mathrm{O}$ evaporation over the cultivation period.

The specific growth rate $\mu_{\max }$ was determined graphically from the curve of the logarithmic mean OTR values for each cultivation experiment. The specific OTR $\mathrm{max}_{\text {was }}$ walculated by dividing the measured OTR $\mathrm{Omax}_{\max }$ by the dry biomass concentration at the corresponding time point.

\section{Determination of oleanolic and ursolic acid}

After biomass determination, the freeze dried biomass was extracted and subjected to HPLC analysis as described previously (Kümmritz et al. 2014). The OA and/or UA concentrations of some samples were below the limit of detection $\left(2.5 \mu \mathrm{g} \mathrm{ml}^{-1}\right)$. For the calculation of the OA and UA contents the value of $2.5 \mu \mathrm{g} \mathrm{ml}^{-1}$ was used and the values were marked with ' $<$ ' in Table 5. The relative standard deviation for the entire extraction and HPLC analysis process was $14.95 \%$ for OA and $14.19 \%$ for UA due to sample heterogeneity arising from cell aggregation (6 replicate extraction and analyses of a single biomass sample). Two parameters were used to assess the production of OA and UA by the different suspension cultures: the content per unit DW ( $\mu \mathrm{g} \mathrm{g}^{-1}$ or $\left.\mathrm{mg} \mathrm{g}^{-1}\right)$ and the volumetric yield (the content multiplied by the biomass concentration for the sample at the same time point, given in $\mathrm{mg}^{-1}$ ).

\section{Analysis of filtrated suspension media}

The conductivity and $\mathrm{pH}$ of the filtrated media were determined directly after sampling at $25^{\circ} \mathrm{C}$. After enzyme inactivation at $80{ }^{\circ} \mathrm{C}(15 \mathrm{~min})$ in a water bath, the filtrate was stored at $-20{ }^{\circ} \mathrm{C}$ until required. Sample preparation and quantification of sucrose, glucose and fructose by HPLC were performed as previously reported (Geipel et al. 2014). The nitrate content was analysed using the photometric LCK-339-Kit (Hach Lange, Düsseldorf, Germany). 


\section{Results and discussion}

\section{Selection of callus lines for establishing suspension cultures}

Callus was successfully induced from young leaves and leaf stems of three different Salvia species (S. officinalis, S. virgata and $S$. fruticosa) in media containing several combinations of PGR's. The calli were very heterogeneous in terms of their production of target metabolites, growth behaviour, morphology and colour. The colour of the callus seems to be species-specific because it did not vary visibly between different PGR combinations. Calli of $S$. officinalis and $S$. fruticosa were bright yellow, while those of $S$. virgata were more greyish.

Four callus morphologies were observed for all Salvia species: a) round-shaped and very compact callus, b) mixtures of compact and friable callus, c) friable callus and d) mixtures of friable and very soft callus with a pulpy consistency (Fig. 1).

The 71 callus lines that produced sufficient biomass for screening were analysed for their OA and UA contents (Table 2). The determined minimum, median and maximum values for OA and UA contents differed strongly between the three tested Salvia species.

S. fruticosa lines exhibited relatively strong OA and UA production, represented by higher median values (642 $\mu \mathrm{g} \mathrm{g}^{-1}$ for OA and $927 \mu \mathrm{g} \mathrm{g}^{-1}$ for UA). S. virgata had a much lower median value for OA and UA than $S$. fruticosa followed by S. officinalis. Comparing the median OA and UA contents for the calli (Table 2) with the minimum and maximum values that have been reported for intact plants (Table 1), the OA and UA outputs of the calli ranged from 0.4 to $10.9 \%$ of those for intact plants. The growth behaviour of the callus (in terms of their AGG values) is shown in Table 2. S. fruticosa callus lines exhibited stronger growth than the other two Salvia species, as indicated by their much higher minimal $(24 \%$ vs. $<10 \%)$ and median AGG values. The influence of PGR's on callus growth and $\mathrm{OA} / \mathrm{UA}$ production remained unconsidered in this work. 
Finally, eleven lines of the heterogeneous pool of screened Salvia calli were selected for the establishment of suspension cultures (Table 3). The criteria for selection were a high content of the target metabolites OA and UA, acceptable growth behaviour and a noncompact morphology.

Preliminary experiments revealed that the medium used for callus induction was not appropriate for promoting the growth of suspension cultures (data not shown). Therefore, the selected calli were transferred from the solid induction media (MS medium containing various auxin/cytokinin combinations) to liquid LS medium supplemented with $0.2 \mathrm{mg} \mathrm{l}^{-1}$ 2,4-D only. This medium has previously been used successfully to establish cell suspension cultures of various plant species (Gyurkovska et al. 2011; Pavlov et al. 2005; Pavlov et al. 2000). After several weeks of cultivation six lines adapted successfully to the new medium and were studied further using RAMOS to characterise their physiology (these lines are listed in Table 3 and highlighted with a dark background). Lines that did not adapt to the new medium were not used in subsequent experiments.

\section{Online data of established suspension cultures obtained in RAMOS}

Each suspension culture has its own growth cycle, so samples taken from different cultures on any given day of cultivation will not necessarily represent the same growth phase and thus may not be comparable. Therefore, respiration data gathered using RAMOS were used to characterise the growth of each culture and to establish schedules for offline sampling such that the samples from each individual suspension culture reflected the same phases of growth.

In general, the measured $\mathrm{OTR}_{\max }$ values for the suspension cultures ranged from $2.0-$ $4.0 \mathrm{mmol} \mathrm{l}^{-1} \cdot \mathrm{h}^{-1}$, while their specific OTR $\max$ values were between 0.17 and $0.38 \mathrm{mmol} \mathrm{l}^{-}$ ${ }^{1} \cdot \mathrm{h}^{-1}$. Assuming that the OTR is approximately equal to the oxygen uptake rates (OUR), the specific OTR $\mathrm{O}_{\max }$ values of the Salvia suspension cultures are comparable to or perhaps 
slightly lower than the values reported in the literature for other plant suspension cultures, which range from 0.27 to $0.59 \mathrm{mmol} \mathrm{g}^{-1} \cdot \mathrm{h}^{-1}$ (Taticek et al. 1991). A sunflower suspension culture that was cultivated under the conditions used in this work exhibited a similar $\mathrm{OTR}_{\max }$ value of $2 \mathrm{mmol} \mathrm{l}^{-1} \cdot \mathrm{h}^{-1}$ (Geipel et al. 2013).

Fig. 2 shows illustrative OTR-curves for two Salvia suspension cultures: $\mathrm{S}_{\text {off_a }}$ and $\mathrm{S}_{\text {fru_a }}$ (for species and line names see Table 3). After inoculation, the OTR values for both suspension cultures were around $0.6 \mathrm{mmol} \mathrm{l}^{-1} \cdot \mathrm{h}^{-1}$, which represents their respiratory activity during the lag-phase. Around two days later, the OTR curves increased due to higher metabolic activity associated with cell growth and division, and the cultures` OT values increased strongly. The OTR curves for both suspension cultures peaked after approximately 9 days of cultivation (Fig. 2a-1 and b-1), after which their OT curves started sloping. This corresponds to the beginning of the transition phase that denotes a reduction in growth activity. Then the OTR decreased further, indicating that the culture had entered the stationary phase.

The decrease in the OTR values on day $10\left(\mathrm{~S}_{\text {off_a }}\right)$ or $11\left(\mathrm{~S}_{\text {fru_a }}\right)$ was probably due to the depletion of sugars within the medium (Fig. 2a-3 and b-3). As shown in Fig. 2 a-3 and b-3, the suspension cultures had completely hydrolysed the original carbon source (sucrose) after 3 - 4 days of cultivation into glucose and fructose, which are metabolized simultaneously. However, in keeping with results observed for several other plant cell suspension cultures, the glucose was taken up more quickly. This may be due to the higher affinity of the hexose-transporter in the plasma-membrane for glucose (Krook et al. 2000). After 10 days, all of the sugar in the medium had been depleted in the case of $\mathrm{S}_{\text {off }}$ a (Fig. 2a-3) and after 11 days, only a small amount of fructose remained in the case of $\mathrm{S}_{\text {fru_a }}$ (Fig. 2b-3). These effects were most probably not due to nitrogen limitation because the final offline samples from all cultures had residual nitrate concentration in excess of $0.7 \mathrm{~g} \mathrm{l}^{-1}$ 
(the initial values was $2.4 \mathrm{~g} \mathrm{l}^{-1}$ ). The OTR curves of the screened cultures all had different forms and scales, even when considering cultures from the same species, highlighting the different growth behaviours of the suspension cultures. As demonstrated by the results for the $S_{\text {off_a }}$ and $S_{\text {fru_a }}$ lines, the increase in OTR was relatively linear in some cases (e.g $S_{\text {off_a }}$, Fig. 2a-1) and almost exponential in others (e.g. Sfru_a, Fig. 2b-1). ). In most cases, the OTR only remained at the OTR $\max$ value for a relatively short period of time (as was observed for $\mathrm{S}_{\text {off_a }}$ ) but some cultures exhibited near-maximal OTR values for multiple days (e.g. $\mathrm{S}_{\text {fru_a }}$ ). A higher rate of agitation supports the $\mathrm{O}_{2}$-transfer within a culture (Maier and Büchs 2001). However, the growth patterns of cultures grown under agitation at 150 rpm did not differ from those for cultures grown at $110 \mathrm{rpm}$, indicating that $\mathrm{O}_{2}$-transfer was not growth-limiting at $110 \mathrm{rpm}$. Extended OTR plateaus have previously been observed for a sunflower suspension culture but an investigation of this effect revealed, that an $\mathrm{O}_{2}$-limitation was not responsible (Geipel et al. 2013). It therefore seems that the occurrence of extended periods when the OTR for the Salvia suspension cultures was close to their $\mathrm{OTR}_{\max }$ was due to an as yet unidentified factor rather than growth limitation because of $\mathrm{O}_{2}$-transfer or inadequate supply of carbon.

The RQ values for the tested cultures (Fig. 2a-1 and b-1) were relatively constant, ranging from 1.1 to 1.2 at the start of cultivation and declining to around 1.0 when the OTR stagnated or decreased. Cultures that are only respiring would be expected to have an RQ of 1.0, which seems to be the case when the growth activity of the cells is reduced or stopped. To the best of our knowledge no data are available in the literature on typical RQ values during plant cell cultivation. Because the molecular formula (primary elemental composition) of the Salvia cultures ' biomass used in this work is unknown, it is not possible to establish theoretical predictions of their RQ. Consequently, it is also impossible to analyse or explain any potential variation or increases in the RQ during the early stages of cultivation. Further investigations into the RQ values, growth, and secondary metabolite 
production of Salvia cell suspension cultures might enable the identification of useful indicators for following these processes and of transition points between different metabolic phases such as the switch from primary to secondary metabolite production.

\section{Growth behaviour of the established suspension cultures}

The online OTR data were used to assess the growth of the suspension cultures, which means the calculation of their specific growth rates because the offline data set based on biomass sampling was too small. The specific growth rate $\mu_{\max }$ is the most useful parameter for characterising the suspension-specific growth behaviour because it is independent of the lag-phase (Table 4). The duration of the lag-phase can be influenced by inoculum size and by the initial ratio of life to dead cells in the cultures, which was not determined in this work.

The growth behaviour $\left(\mu_{\max }\right)$ of the cultures fluctuated between the cultivations. All $\mu_{\max }$ values independent from the applied shaking frequency were taken into account in Table 4 because the $\mu_{\max }$ values for cultures grown at $150 \mathrm{rpm}$ were within the RSD of the values obtained at $110 \mathrm{rpm}$ or no clear tendency to better or worse growth at $150 \mathrm{rpm}$ compared to $110 \mathrm{rpm}$ is apparent; that is to say, there was no appreciable difference between the growth behaviours observed at 110 and $150 \mathrm{rpm}$. The calculated $\mu_{\max }$ values ranged from 0.16 to $0.53 \mathrm{~d}^{-1}$ and are comparable to those reported for suspension cultures of Nicotiana tabacum $\left(0.47 \mathrm{~d}^{-1}\right)$ or Catharanthus roseus $\left(0.33 \mathrm{~d}^{-1}\right)$ (van Gulik et al. 1992).

In general, the mean $\mu_{\max }$ values for the $S$. virgata suspension cultures $\left(>0.34 \mathrm{~d}^{-1}\right)$ were greater than those for S. officinalis or S. fruticosa cultures, which were both around $0.20 \mathrm{~d}^{-}$

${ }^{1}$. The suspension culture with the highest mean $\mu_{\max }\left(\approx 0.40 \mathrm{~d}^{-1}\right) \mathrm{S}_{\mathrm{vir} \_}$while that with the lowest specific growth rate was $\mathrm{S}_{\text {off_b }}\left(0.17 \mathrm{~d}^{-1}\right)$. The very slow growth of this latter suspension culture makes it unsuitable as a production system. Therefore, no further 
experiments were performed with $\mathrm{S}_{\text {off_b }}$. The maximum dry biomass concentrations for all investigated suspension cultures ranged from 10.8 to $15.7 \mathrm{~g} \mathrm{l}^{-1}$.

Although the suspensions were all grown on identical media, they differed strongly in terms of their growth rates and aggregation behaviour, clearly demonstrating the need for detailed screening.

\section{The kinetics of product formation in established suspension cultures}

Fig. $3 \mathrm{a}$ and $3 \mathrm{~b}$ show illustrative online OTR data acquired using RAMOS and product content data (OA, UA) determined via offline sampling for the $\mathrm{S}_{\text {off_a }}$ and $\mathrm{S}_{\text {fru_a }}$ cultures grown at a shaking frequency at $110 \mathrm{rpm}$. In both cases, the OA and UA contents remain more or less constant and may even decline slightly at the start of cultivation and during the early stages of the growth phase (Fig. 3a and b). The OA and UA content within dry biomass increased in the second half of the growth phase or at the start of the transitional phase (which occur after 11 and 7 days of cultivation, respectively).

Because RAMOS can only accommodate a limited number of measuring flasks, a standard shake flask experiment with daily sampling was performed using the $\mathrm{S}_{\text {off_a }}$ suspension culture in order to acquire more detailed information on product formation (Fig. 3c). During the first day, there was an increase in the culture's triterpenic acid content that may have been induced by new osmotic conditions presented by the fresh media during inoculation and it is known that osmotic stress can have eliciting effects (Kim et al. 2001). The cultures OA and UA content did not change appreciably over the next three days, which is consistent with culture being in the lag phase of growth, when it would not be expected to produce secondary metabolites. After the fourth day of cultivation the OA and UA content started to decrease strongly when the cultures began growing intensively. This could be caused by a dilution effect arising from the increase in the culture`s biomass and from degradation of existing triterpenic acids. After the sixth day, triterpenic acid 
production resumed and the levels of OA and UA in the biomass samples rose continuously until day 10 of cultivation. Between days 8 and 10, growth activity stagnated but the production of the target metabolites continued until the culture`s OA and UA contents were identical to those at the start of cultivation. This is consistent with the use of a 10 day old inoculum for the start of the experiments. Based on this increasing trend, one might expect the OA and UA content to increase further on day 11 and beyond that and indeed, after 11 days of $\mathrm{S}_{\text {off_a }(1)}$ cultivation using RAMOS, the product content was greater than that observed at the start of the experiment (Fig. 3a). In general, the OA and UA contents determined from the samples collected during the cultivation of $\mathrm{S}_{\text {off }} \mathrm{a}$ in the shake flasks confirmed the trends observed in the RAMOS data. Similar data on the relationship between growth and the production of OA and UA (Fig. 3) have been reported for a suspension culture of Perilla frutescens (Wang et al. 2004). However, reductions in UA levels during the later stages of the growth phase have been observed for two other suspension cultures of S. officinalis (Bolta et al. 2000). Overall, our results indicate that the production of $\mathrm{OA}$ and $\mathrm{UA}$ is maximised during the late stage of the growth phase and the stationary phase. Prolonging the stationary phase by adding further sucrose to the medium may thus enhance OA and UA production.

\section{Assessment of OA and UA production in established suspension cultures}

To assess the production of OA and UA of the investigated suspension cultures the maximal measured product contents and the corresponding volumetric yields were considered (Table 5).

The mean values for $\mathrm{OA}_{\max }$ and $\mathrm{UA}_{\max }$ contents for the suspension cultures ranged from $0.29-4.08 \mathrm{mg} \mathrm{OA} \mathrm{g}^{-1} \mathrm{DW}$ and $0.12-3.23 \mathrm{mg} \mathrm{UA} \mathrm{g}^{-1} \mathrm{DW}$, respectively. The highest triterpene contents and volumetric yields were obtained from suspension cultures of $S$. officinalis $\left(\mathrm{S}_{\text {off_a }}, \mathrm{S}_{\text {off_b }}\right)$ and $S$. fruticosa $\left(\mathrm{S}_{\text {fru_a }}\right)$. The $\mathrm{S}_{\text {fru_a }}$ and $\mathrm{S}_{\text {off_a }}$ cultures also had the 
lowest RSD`s for these variables $(22-39 \%)$, indicating that their output was relatively stable. While, growth and secondary metabolite formation can be negatively correlated in some cases (Hagendoorn et al. 1997) we did not observe any correlation between $\mu_{\max }$ and the maximum cumulative OA and UA content for most of the cultures (data not shown). Only the data for $\mathrm{S}_{\text {off_b }}$ are somewhat consistent with this hypothesis because this culture exhibited very weak growth and the highest product contents. However, as previously mentioned, this culture grew very slowly and was therefore only studied in a single RAMOS cultivation experiment. Consequently, no firm conclusion can be drawn from this result.

The OA and UA contents of the screened Salvia suspension cultures are comparable to those reported for other in vitro cultures (Muffler et al. 2011; Georgiev et al. 2011; FeriaRomero et al. 2005).

Interestingly, the OA:UA ratio differs among the three species and depends on the type of in vitro culture considered (callus or suspension). There was no clear species-specific OA:UA ratio. However, the $S$. fruticosa suspension culture $\left(\mathrm{S}_{\mathrm{fru} \_\mathrm{a}}\right)$ and corresponding intact plants (Table 1) have a low OA:UA ratio and produce much more UA than OA. The suspension cultures of $S$. officinalis and $S$. virgata often produce equal amounts of both triterpenic acids.

The OA and UA concentrations within the screened suspension cultures were substantially lower than those seen in intact plants (Table 1). S fru $_{-}$p produces around $14 \%$ as much OA and $10 \%$ as much UA as has been reported for S. fruticosa. The plants used to induce the callus cultures of $S$. officinalis and $S$. virgata in this study were obtained from the same seed batch as the plants used by Kümmritz et al. (2014), so the OA and UA contents for cultures of these species will only be compared to those authors` results. The OA and UA content of the S. officinalis suspension culture $\mathrm{S}_{\text {off_a }}$ were $54 \%$ and $11 \%$ of the values 
observed for intact plants. $\mathrm{S}_{\mathrm{vir} \_}$, the most productive $S$. virgata suspension culture accumulated $24 \%$ and $5 \%$ of the OA and UA contents measured for intact $S$. virgata plants.

\section{Final assessment of the screened suspension cultures and prospects for future}

\section{development}

Based on their growth behaviour and productivity, the most appropriate suspension cultures for further investigation and optimisation are $\mathrm{S}_{\text {fru_a }}$, $S_{\text {vir_c }}$, and $S_{\text {off_a }}$. The specific growth rates of $S_{\text {fru_a }}$ and $S_{\text {off_a }}$ were lower than those for the $S_{\text {vir }}$ suspensions $\left(0.2 \mathrm{~d}^{-1}\right.$ vs. $>$ $0.3 \mathrm{~d}^{-1}$, compare Table 4) but both cultures exhibited good growth stability (see the RSD values listed in Table 4) and, together with $\mathrm{S}_{\text {off }}$ b, they gave the highest volumetric yields of

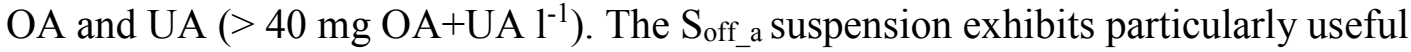
behaviour with respect to aggregate formation because it is very homogenous and consists of aggregates smaller than $1 \mathrm{~mm}$. The $S_{\text {fru_a }}$ and $S_{\text {vir_c }}$ suspensions are more heterogeneous and contain aggregates up to around $5 \mathrm{~mm}$ in diameter but also have high proportions of very small aggregates. Regardless, they merit further investigation.

The OA and UA contents per unit DW of these three cultures were at least $10 \%$ of those seen in the corresponding intact plants. These levels could potentially be increased further via media optimisation, elicitation (Marchev et al. 2014) and the use of appropriate cultivation systems (Georgiev et al. 2013). In addition, the establishment of cryopreservation protocols that would preserve the biosynthetic potential of the cultures would be desirable (Mustafa et al. 2011) because it is known that problems with the longterm stability of growth and productivity can occur with plant in vitro cultures (Smetanska 2008). 
Acknowledgement: We thank Eva-Maria Kneschke, Héctor Gómez Morales and Kristin Halbauer for technical support; Jörg-Uwe Ackermann, Christian Löser, Katja Geipel and Maria Lisa Socher for helpful and stimulating discussions. This study was supported financially by German Academic Exchange Service (DAAD, PhD Fellowship) and German Research Foundation (DFG, project ID: BL345/10-1). 


\section{References}

Anderlei T, Büchs J (2001) Device for sterile online measurement of the oxygen transfer rate in shaking flasks. Biochem Eng J 7:157-162. doi: 10.1016/S1369703X(00)00116-9

Anderlei T, Zang W, Papaspyrou M, Büchs J (2004) Online respiration activity measurement (OTR, CTR, RQ) in shake flasks. Biochem Eng J 17:187-194. doi: $10.1016 / \mathrm{S} 1369-703 \mathrm{X}(03) 00181-5$

Bolta Ž, Baričevič D, Bohanec B, Andrenše S (2000) A preliminary investigation of ursolic acid in cell suspension culture of Salvia officinalis. Plant Cell Tissue Organ Cult 62:57-63. doi: 10.1023/A:1006498431099

Feria-Romero I, Lazo E, Ponce-Noyola T, et al. (2005) Induced accumulation of oleanolic acid and ursolic acid in cell suspension cultures of Uncaria tomentosa. Biotechnol Lett 27:839-843. doi: 10.1007/s10529-005-6215-7

Geipel K, Socher ML, Haas C, et al. (2013) Growth kinetics of a Helianthus annuus and a Salvia fruticosa suspension cell line: Shake flask cultivations with online monitoring system. Eng Life Sci 13:593-602. doi: 10.1002/elsc.201200148

Geipel K, Song X, Socher ML, et al. (2014) Induction of a photomixotrophic plant cell culture ofHelianthus annuusand optimization of culture conditions for improved $\alpha$ tocopherol production. Appl Microbiol Biotechnol 98:2029-2040. doi: DOI $10.1007 / \mathrm{s} 00253-013-5431-7$

Georgiev MI, Eibl R, Zhong J-J (2013) Hosting the plant cells in vitro: recent trends in bioreactors. Appl Microbiol Biotechnol 97:3787-3800. doi: 10.1007/s00253-013$4817-\mathrm{x}$

Georgiev V, Marchev A, Haas C, et al. (2011) Production of oleanolic and ursolic acids by callus cultures of Salvia tomentosa Mill. Biotechnol Biotechnol Equip 25:34-38. doi: 10.5504/bbeq.2011.0129

Van Gulik WM, ten Hoopen HJG, Heijnen JJ (1992) Kinetics and stoichiometry of growth of plant cell cultures of Catharanthus roseus and Nicotiana tabacum in batch and continuous fermentors. Biotechnol Bioeng 40:863-874. doi: 10.1002/bit.260400802

Gyurkovska V, Alipieva K, Maciuk A, et al. (2011) Anti-inflammatory activity of Devil's claw in vitro systems and their active constituents. Food Chem 125:171-178. doi: 10.1016/j.foodchem.2010.08.056

Hagendoorn MJM, Jamar DCL, Meykamp B, van der Plas LHW (1997) Cell division versus secondary metabolite production in Morinda citrifolia cell suspensions. J Plant Physiol 150:325-330. doi: 10.1016/S0176-1617(97)80128-9

Janicsák G, Veres K, Zoltán Kakasy A, Máthé I (2006) Study of the oleanolic and ursolic acid contents of some species of the Lamiaceae. Biochem Syst Ecol 34:392-396. doi: 16/j.bse.2005.12.004 
Karam NS, Jawad FM, Arikat NA, Shibl RA (2003) Growth and rosmarinic acid accumulation in callus, cell suspension, and root cultures of wild Salvia fruticosa. Plant Cell Tissue Organ Cult 73:117-121. doi: 10.1023/A:1022806420209

Kensy F, Engelbrecht C, Büchs J (2009) Scale-up from microtiter plate to laboratory fermenter: evaluation by online monitoring techniques of growth and protein expression in Escherichia coli and Hansenula polymorpha fermentations. Microb Cell Factories 8:68. doi: 10.1186/1475-2859-8-68

Kim S-I, Choi H-K, Kim J-H, et al. (2001) Effect of osmotic pressure on paclitaxel production in suspension cell cultures of Taxus chinensis. Enzyme Microb Technol 28:202-209. doi: 10.1016/S0141-0229(00)00292-1

Kintzios S, Nikolaou A, Skoula M (1999) Somatic embryogenesis and in vitro rosmarinic acid accumulation in Salvia officinalis and S. fruticosa leaf callus cultures. Plant Cell Rep 18:462-466. doi: 10.1007/s002990050604

Krook J, Vreugdenhil D, van der Plas LHW (2000) Uptake and phosphorylation of glucose and fructose in Daucus carota cell suspensions are differently regulated. Plant Physiol Biochem 38:603-612. doi: 10.1016/S0981-9428(00)00776-2

Kümmritz S, Haas C, Pavlov AI, et al. (2014) Determination of triterpenic acids and screening for valuable secondary metabolites in Salvia sp. suspension cultures. Nat Prod Commun 9:17-20.

Liu J (2005) Oleanolic acid and ursolic acid: Research perspectives. J Ethnopharmacol 100:92-94. doi: 10.1016/j.jep.2005.05.024

Liu J (1995) Pharmacology of oleanolic acid and ursolic acid. J Ethnopharmacol 49:57-68. doi: 10.1016/0378-8741(95)90032-2

Maier U, Büchs J (2001) Characterisation of the gas-liquid mass transfer in shaking bioreactors. Biochem Eng J 7:99-106. doi: 16/S1369-703X(00)00107-8

Marchev A, Haas C, Schulz S, et al. (2014) Sage in vitro cultures: a promising tool for the production of bioactive terpenes and phenolic substances. Biotechnol Lett 36:211221. doi: $10.1007 / \mathrm{s} 10529-013-1350-\mathrm{Z}$

Martin R, Fausten G, Bischoff F (2009) Screening verschiedener Arten auf ihren Gehalt an den Triterpenen Ursol- und Oleanolsäure. J Med Spice Plants 14:37-43.

Mathur S, Shekhawat GS (2013) Establishment and characterization of Stevia rebaudiana (Bertoni) cell suspension culture: an in vitro approach for production of stevioside. Acta Physiol Plant 35:931-939. doi: 10.1007/s11738-012-1136-2

Muffler K, Leipold D, Scheller M-C, et al. (2011) Biotransformation of triterpenes. Process Biochem 46:1-15. doi: 16/j.procbio.2010.07.015

Mustafa NR, de Winter W, van Iren F, Verpoorte R (2011) Initiation, growth and cryopreservation of plant cell suspension cultures. Nat Protoc 6:715-742. doi: 10.1038 /nprot.2010.144 
Pavlov A, Werner S, Ilieva M, Bley T (2005) Characteristics of Helianthus annuus Plant Cell Culture as a Producer of Immunologically Active Exopolysaccharides. Eng Life Sci 5:280-283. doi: 10.1002/elsc.200420074

Pavlov AI, Ilieva MP, Panchev IN (2000) Nutrient Medium Optimization for Rosmarinic Acid Production by Lavandula vera MM Cell Suspension. Biotechnol Prog 16:668670. doi: $10.1021 / \mathrm{bp} 000041 \mathrm{z}$

Smetanska I (2008) Production of Secondary Metabolites Using Plant Cell Cultures. Adv. Biochem. Eng. Biotechnol. pp 187-228

Taarit MB, Msaada K, Hosni K, Marzouk B (2011) Physiological changes and essential oil composition of clary sage (Salvia sclarea L.) rosette leaves as affected by salinity. Acta Physiol Plant 33:153-162. doi: 10.1007/s11738-010-0532-8

Taticek RA, Moo-Young M, Legge RL (1991) The scale-up of plant cell culture: Engineering considerations. Plant Cell Tissue Organ Cult 24:139-158. doi: 10.1007/BF00039742

Ullisch DA, Müller CA, Maibaum S, et al. (2012) Comprehensive characterization of two different Nicotiana tabacum cell lines leads to doubled GFP and HA protein production by media optimization. J Biosci Bioeng 113:242-248. doi: 10.1016/j.jbiosc.2011.09.022

Wang JW, Xia ZH, Chu JH, Tan RX (2004) Simultaneous production of anthocyanin and triterpenoids in suspension cultures of Perilla frutescens. Enzyme Microb Technol 34:651-656. doi: 10.1016/j.enzmictec.2004.02.004 
Table 1 Reported oleanolic and ursolic acid contents for the three sage species examined in this work.

\begin{tabular}{|c|c|c|c|}
\hline Species & $\begin{array}{l}\text { OA } \\
{\left[\mathrm{mg} \mathrm{g}^{-1} \mathrm{DW}\right]}\end{array}$ & $\begin{array}{l}\text { UA } \\
{\left[\mathrm{mg} \mathrm{g}^{-1} \mathrm{DW}\right]}\end{array}$ & Reference* \\
\hline \multirow[t]{4}{*}{ S. officinalis } & 6.5 & 18.6 & (Janicsák et al. 2003) \\
\hline & 15.6 & 38.3 & (Janicsák et al. 2006) \\
\hline & $5.6-19.7$ & $9.7-26.6$ & (Martin et al. 2009) \\
\hline & 7.5 & 22.5 & (Kümmritz et al. 2014) \\
\hline \multirow[t]{2}{*}{ S. fruticosa } & 0.011 & - & $\begin{array}{l}\text { calculated from (El-Sayed } \\
\text { et al. 2001) }\end{array}$ \\
\hline & 10.8 & 31.1 & (Kümmritz et al. 2014) \\
\hline \multirow[t]{2}{*}{ S. virgata } & 2.9 & 3.5 & (Janicsák et al. 2006) \\
\hline & 5.5 & 27.5 & (Kümmritz et al. 2014) \\
\hline
\end{tabular}


Table 2 Triterpene content $(\mathrm{RSD}=14.95 \%$ for OA and $14.19 \%$ for UA) of the callus lines and growth behaviour of the callus lines expressed in terms of the area gained by growth $(\mathrm{AGG}, \mathrm{RSD}=12.9 \%)$ for the three used sage species. Minimum, maximum and median values for both parameters are presented.

\begin{tabular}{|c|c|c|c|c|c|c|c|c|c|c|}
\hline \multirow{3}{*}{ Species } & \multirow{3}{*}{ No. of lines } & \multicolumn{6}{|c|}{ Triterpene content $\left[\mu g^{-1} \mathrm{DW}\right]$} & \multicolumn{3}{|c|}{ Growth behaviour - AGG [\%] } \\
\hline & & \multicolumn{2}{|c|}{ Min } & \multicolumn{2}{|c|}{ Median } & \multicolumn{2}{|c|}{ Max } & \multirow[t]{2}{*}{ Min } & \multirow[t]{2}{*}{ Median } & \multirow[t]{2}{*}{$\operatorname{Max}$} \\
\hline & & $\boldsymbol{O A}$ & $\boldsymbol{U A}$ & $\boldsymbol{O A}$ & $\boldsymbol{U A}$ & $\boldsymbol{O A}$ & $\boldsymbol{U A}$ & & & \\
\hline S. officinalis & 57 & 0 & 0 & 80 & 215 & 1901 & 4294 & 4.6 & 64.9 & 198.9 \\
\hline S. virgata & 9 & 49 & 35 & 384 & 292 & 1350 & 1050 & 10.1 & 37.5 & 99.5 \\
\hline S. fruticosa & 5 & 378 & 762 & 642 & 927 & 825 & 2425 & 23.9 & 70.0 & 149.6 \\
\hline
\end{tabular}


Table 3 Characteristics of the callus lines that were selected for the establishment of suspension cultures. Lines that adapted successfully to the indicated liquid LS medium and grew are highlighted with a grey background.

$\begin{array}{llll}\text { Line } & \text { Plant growth regulators } & \text { Triterpene content } & \text { Growth } \\ \text { name } & \text { used for callus induction } & & \text { behaviour }\end{array}$

$$
\left[\mathrm{mg} \mathrm{l}^{-1}\right] \quad\left[\mu_{g^{-1}} \mathrm{DW}\right] \quad \text { AGG }[\%]
$$

auxin cytokinin $\quad$ OA UA

\section{S. officinalis}

\begin{tabular}{|c|c|c|c|c|c|}
\hline $\mathrm{S}_{\text {off_a }}$ & 2,4-D: 0.5 & Kin: 0.5 & 97 & 164 & 69 \\
\hline $\mathrm{S}_{\text {off_b }}$ & 2,4-D: 1 & Zea: 0.5 & 1517 & 1026 & 38 \\
\hline $\mathrm{S}_{\text {off_c }}$ & 2,4-D: 1 & BAP: 0.5 & 1206 & 814 & 41 \\
\hline $\mathrm{S}_{\text {off_d }}$ & NAA: 1 & Kin: 1 & 178 & 1385 & 14 \\
\hline $\mathrm{S}_{\text {off_e }}$ & Pic: 0.5 & Kin: 0.5 & 677 & 207 & 57 \\
\hline $\mathrm{S}_{\text {off_f }}$ & Pic: 1 & BAP: 1 & 32 & 139 & 94 \\
\hline
\end{tabular}

$\begin{array}{lccccc}\text { S }_{\text {vir_a }} & \text { 2,4-D: } 1 & - & 438 & 605 & 99 \\ \text { S }_{\text {vir_b }} & \text { 2,4-D: } 1 & \text { Kin: } 1 & 356 & 288 & 30 \\ \text { S }_{\text {vir_c }} & \text { 2,4-D: } 0.5 & \text { Zea: } 0.5 & 476 & 354 & 32 \\ \text { S. fruticosa } & & & & & \end{array}$

\begin{tabular}{llcccc} 
S fru_a $_{-}$ & 2,4-D: 1 & Zea: 0.5 & 825 & 2425 & 70 \\
\hline Sfru_b & 2,4-D: 0.5 & Zea: 0.5 & 378 & 762 & 150
\end{tabular}


Table 4 Specific growth rate $\left(\mu_{\max }\right)$ values for the screened suspensions $(\mathrm{n}=3)$ based on two measurements for a shaking frequency of $110 \mathrm{rpm}$ and one for a shaking frequency at $150 \mathrm{rpm}$. The values for the $S_{\text {off } \_b}(n=1)$ and $S_{\text {fru_a }}(n=2)$ cultures based only on measurements obtained at $110 \mathrm{rpm}$.

\begin{tabular}{lcc}
\hline Suspension & Mean $\boldsymbol{\mu}_{\max }\left[\mathbf{d}^{-\mathbf{1}}\right]$ & RSD [\%] \\
\hline S. officinalis & & 27.9 \\
Soff_a $_{\text {off_b }}$ & 0.20 & - \\
S. virgata $_{\text {off }}$ & 0.17 & \\
Svir_a $_{\text {Sir_b }}$ & 0.40 & 26.9 \\
S $_{\text {vir_c }}$ & 0.34 & 49.8 \\
S. fruticosa & 0.37 & 33.8 \\
S $_{\text {fru_a }}$ & & \\
\hline
\end{tabular}


Table 5 Maximum OA and UA contents per unit DW for each suspension culture and volumetric yields of OA and UA at the corresponding time points. Values below the limit of detection are indicated by the ' $<$ ' symbol; in such cases, the measured sample concentration was assumed to be equal to the limit of detection for the purpose of subsequent calculations. n.d. = not detectable

\begin{tabular}{|c|c|c|c|c|}
\hline \multirow[t]{2}{*}{ Line } & \multicolumn{2}{|c|}{$\begin{array}{c}\text { Maximum triterpene content } \\
{\left[\mathrm{mg} \mathrm{g}^{-1}\right]}\end{array}$} & \multicolumn{2}{|c|}{$\begin{array}{l}\text { Volumetric yield } \\
\qquad\left[\mathrm{mg} \mathrm{l}^{-1}\right]\end{array}$} \\
\hline & OAMax & UAMax & $\mathbf{O A}$ & UA \\
\hline $\mathrm{S}_{\text {off_a }}$ (1) & 1.14 & 1.32 & 17.9 & 20.7 \\
\hline $\mathrm{S}_{\text {off_a }}$ (2) & 1.64 & 1.53 & 26.9 & 25.2 \\
\hline $\mathrm{S}_{\text {off_a }}$ (3) & 1.15 & 0.90 & 18.1 & 14.1 \\
\hline mean & 1.31 & 1.25 & 21.0 & 20.0 \\
\hline $\operatorname{RSD}[\%]$ & 22 & 26 & 25 & 28 \\
\hline $\mathrm{S}_{\text {off_b }}$ & 4.08 & 2.54 & 40.1 & 25.0 \\
\hline mean & - & - & - & - \\
\hline $\operatorname{RSD}[\%]$ & - & - & - & - \\
\hline $\mathrm{S}_{\text {vir_a }}$ (1) & $<0.09$ & $<0.09$ & $<1.3$ & $<1.3$ \\
\hline $\mathrm{S}_{\mathrm{vir} \_\mathrm{a}(2)}$ & $<0.18$ & $<0.21$ & $<1.9$ & $<2.1$ \\
\hline $\mathrm{S}_{\text {vir_a }}$ (3) & 1.93 & 2.28 & 27.7 & 32.6 \\
\hline mean & 0.73 & 0.86 & 10.3 & 12.0 \\
\hline $\operatorname{RSD}[\%]$ & 142 & 144 & 147 & 149 \\
\hline $\mathrm{S}_{\mathrm{vir} \_b(1)}$ & $<0.16$ & n.d. & $<1.9$ & n.d. \\
\hline $\mathrm{S}_{\mathrm{vir} \_\mathrm{b}(2)}$ & 0.55 & 0.36 & 6.5 & 4.3 \\
\hline $\mathrm{S}_{\mathrm{vir} \_\mathrm{b}(3)}$ & $<0.16$ & n.d. & $<1.8$ & n.d. \\
\hline mean & 0.29 & 0.12 & 3.4 & 1.4 \\
\hline
\end{tabular}




\begin{tabular}{lcccc} 
RSD [\%] & 79 & 173 & 80 & 173 \\
\hline $\mathrm{S}_{\text {vir_c (1) }}$ & 1.21 & 1.25 & 17.0 & 17.5 \\
$\mathrm{~S}_{\text {vi_c (2) }}$ & 2.08 & 2.27 & 31.0 & 34.1 \\
$\mathrm{~S}_{\text {vir_c (3) }}$ & 0.64 & 0.53 & 6.9 & 5.7 \\
mean & 1.31 & 1.35 & 18.4 & 19.1 \\
RSD [\%] & 55 & 65 & 67 & 75 \\
\hline $\mathrm{S}_{\text {fru_a (1) }}$ & 1.87 & 4.11 & 16.3 & 35.8 \\
$\mathrm{~S}_{\text {fru_a (2) }}$ & 1.14 & 2.35 & 14.4 & 29.7 \\
mean & 1.50 & 3.23 & 15.3 & 32.8 \\
RSD [\%] & 34 & 39 & 9 & 13 \\
\hline
\end{tabular}




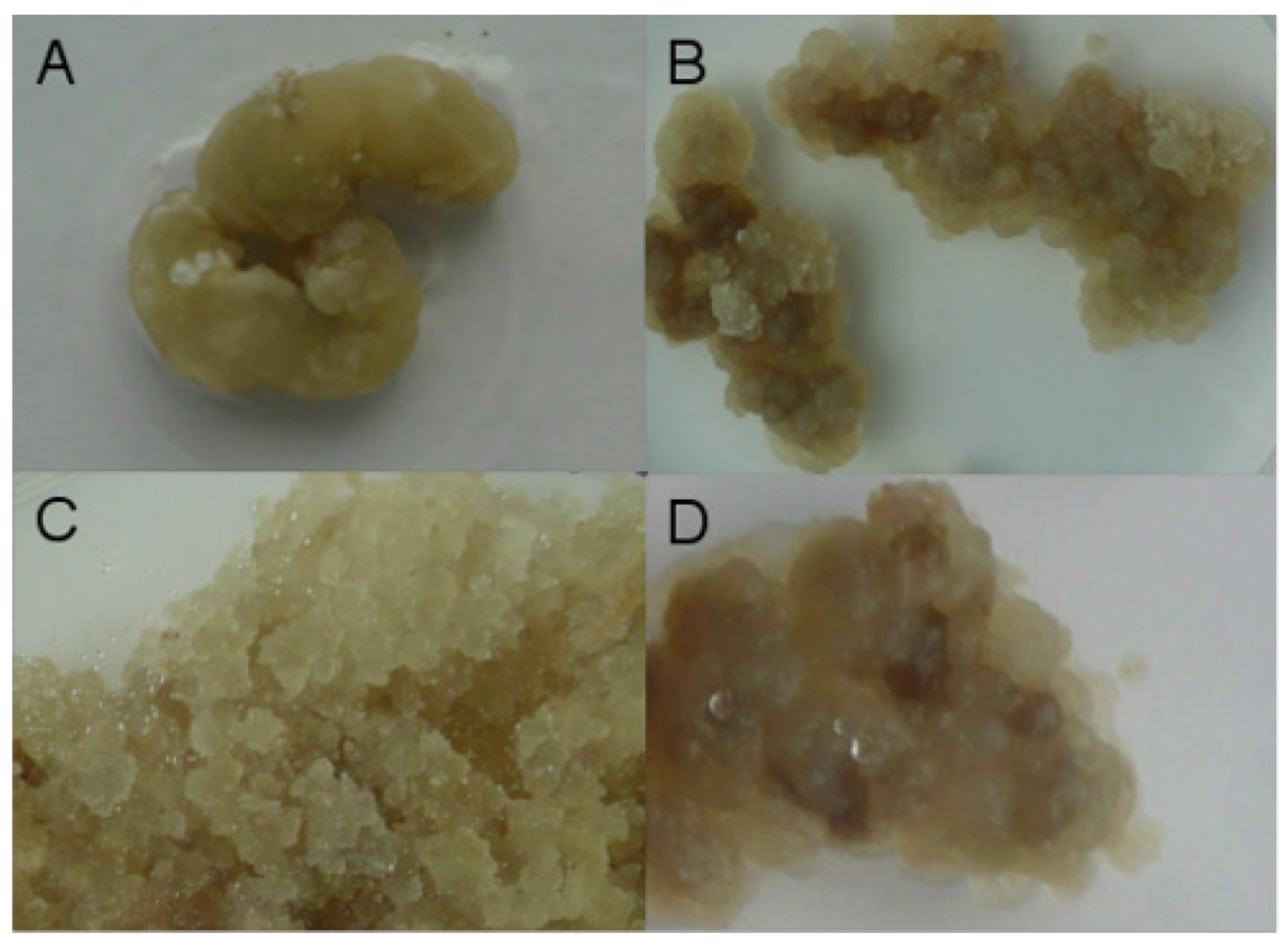

Fig. 1 Morphological properties of calli from the different Salvia species

considered in this work: $\mathbf{a}$ - round-shaped and very compact callus, $\mathbf{b}$ - mixtures

of compact and friable callus, $\mathbf{c}$ - friable callus, $\mathbf{d}$ - friable and very soft callus with a pulpy consistency 

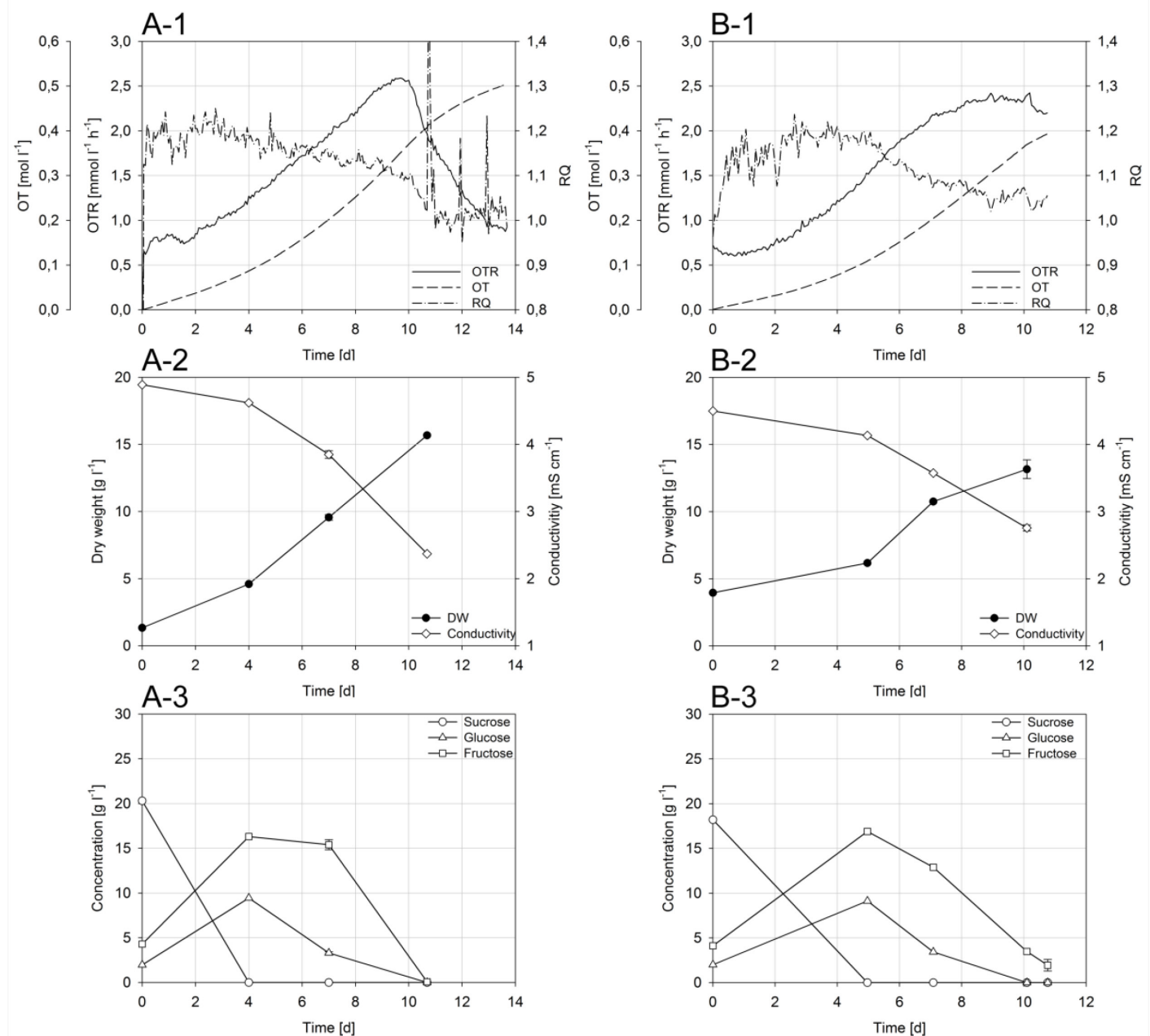

Fig. 2 Representative online (OTR and OT-curves, a-1: Soff_a, b-1: Sfru_a) and offline (DW, a-2: Soff_a, b-2: Sfru_a and sugars, a-3: Soff_a, b-3: Sfru_a) data for two sage suspensions cultivated using the RAMOS at $110 \mathrm{rpm}$ 

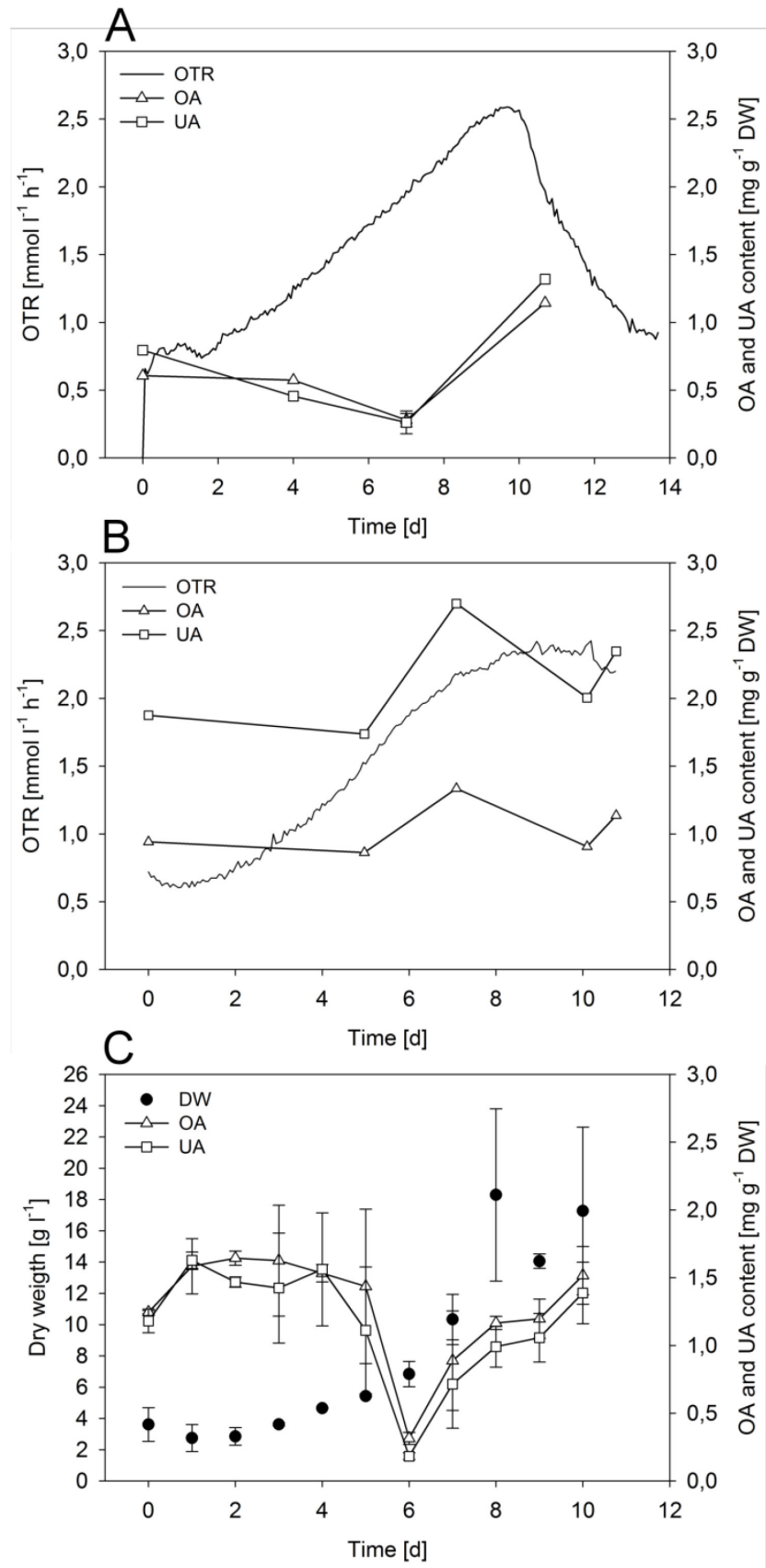

Fig. 3 Representative online (OTR) and offline (OA and UA contents) data for RAMOS cultivations of $\mathrm{S}_{\text {off_a }}(\mathbf{a})$ and $\mathrm{S}_{\text {fru }}$ a $(\mathbf{b})$ at $110 \mathrm{rpm}$ as well as DW measurements and triterpenic acid contents for the $\mathrm{S}_{\text {off_a }}$ suspension culture from a shake flask experiment conducted at $110 \mathrm{rpm}(\mathbf{c})$ 


\section{Author Contributions Statement}

Christiane Haas designed the experiments and wrote the manuscript. KarlChristoph Hengelhaupt performed the experiments. Sibylle Kümmritz contributed to the analysis of OA and UA and the manuscript's preparation. Atanas Pavlov provided scientific support during callus and suspension induction, and was highly involved in the manuscript's preparation and the discussion of the results. Thomas Bley supervised the study. Juliane Steingroewer contributed to the discussion and manuscript preparation. All authors have read and approved the final manuscript. 